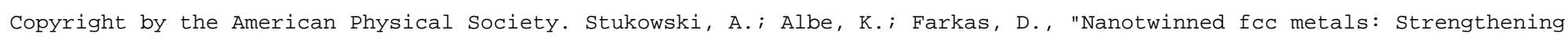
versus softening mechanisms," Phys. Rev. B 82, 224103 DoI: http://dx.doi.org/10.1103/PhysRevB.82.224103

\title{
Nanotwinned fcc metals: Strengthening versus softening mechanisms
}

\author{
A. Stukowski* and K. Albe \\ Institut für Materialwissenschaft, Technische Universität Darmstadt, Darmstadt, Germany \\ D. Farkas \\ Materials Science and Engineering, College of Engineering, Virginia Polytechnical Institute and State University, Holden Hall, \\ Blacksburg, Virginia 24060, USA
}

(Received 24 June 2010; published 8 December 2010)

\begin{abstract}
The strengthening effect of twins in nanocrystalline metals has been reported both in experiment and simulation. While twins are mostly considered as effective barriers to dislocation slip transfer, they can also provide nucleation sites for dislocations or migrate during the deformation process, thereby contributing to plasticity. By comparing twinned and nontwinned samples, we study the effect of twins on the deformation behavior of nanocrystalline $\mathrm{Cu}$ and $\mathrm{Pd}$ using atomistic simulations. While $\mathrm{Cu}$ shows hardening due to the presence of twins, Pd shows the opposite effect. A quantitative dislocation analysis method is applied, which allows to analyze dislocation interactions with twin planes and grain boundaries and to measure dislocation, stacking fault, and twin-boundary densities as functions of strain. A statistical analysis of the occurring dislocation types provides direct evidence for the role of twin boundaries as effective sources for twinning dislocations, which are the reason for the observed softening in some fcc materials. In addition, we discuss how the orientation of the loading direction with respect to the twin planes affects the response of nanotwinned $\mathrm{Cu}$ and Pd.
\end{abstract}

DOI: 10.1103/PhysRevB.82.224103

PACS number(s): 62.25.- g, 61.46.Hk

\section{INTRODUCTION}

Experimental studies show that nanoscale growth twins in ultrafine $\mathrm{Cu}$ can significantly improve mechanical properties as compared to twin-free samples. In tensile tests and nanoindentation experiments, nanotwinned samples with various twin densities exhibit higher yield strength, tensile strength, and hardness as well as ductility. ${ }^{1-3}$ The observed increase in strength with decreasing twin-boundary (TB) distance is comparable to that found for conventional grain-size refinement. That is, nanoscale TBs seem to impart as much strengthening as conventional high-angle grain boundaries (GBs) by blocking dislocation motion ${ }^{1,4}$ and exhibit a HallPetch-type behavior. In contrast to nanocrystalline (nc) $\mathrm{Cu}$ with general GBs, however, nanotwinned $\mathrm{Cu}$ does not lose its tensile ductility when refined to the nanometer regime. In addition, TBs usually exhibit much higher mechanical and thermal stability, and less electric resistivity in comparison to other grain boundaries. ${ }^{4,5}$

The recent experimental work on nanotwinned $\mathrm{Cu}$ (Refs. 1-4 and 6-12) is accompanied by a series of moleculardynamics (MD) studies that investigate the effect of TBs on the deformation behavior. ${ }^{13-24}$ The aim of these simulations is to understand the atomistic mechanisms that give nanotwinned materials their unique properties. In general, the strengthening effect of nanolayered structures is understood to be the result of restricting dislocation motion from one layer to the next. ${ }^{25}$ That is, TBs are conventionally ascribed the role of barriers for dislocation motion. In MD simulations, however, slip transfer of dislocations across TBs has been observed under certain loading conditions and dislocation orientations. ${ }^{16,21,22}$ The overall strengthening effect of nanotwins in $\mathrm{Cu}$ has been confirmed by all published simulation studies.

With only a very few exceptions, ${ }^{13,14,17}$ most work on the effect of growth twins on the mechanical properties of metals have been performed in $\mathrm{Cu}$. Frøseth et al.,${ }^{13,14}$ however, have reported that nanotwinned $\mathrm{Al}$ exhibits enhanced plasticity in constant-stress simulations compared to its twin-free counterpart. This result suggests that other nanotwinned facecentered-cubic (fcc) materials might not show the strengthening effect found for $\mathrm{Cu}$. Jin et al. ${ }^{21,22}$ studied the interaction of single dislocations with a $\mathrm{TB}$ in $\mathrm{Cu}, \mathrm{Ni}$, and $\mathrm{Al}$. They found several possible reactions, being dependent on the material's energy barriers, type of dislocation, and loading condition. For nanotwinned Pd, which is also investigated in the present paper, Kulkarni et al. ${ }^{17}$ predicted a performance based on simulated indentation studies that is very similar to $\mathrm{Cu}$.

Experimental measurements indicate high densities of stacking faults and Shockley partials associated with TBs in nanotwinned $\mathrm{Cu}$ (Ref. 3) after deformation. In addition, a large number of debris was found in transmission electron microscopy (TEM) studies of deformed nanotwinned metals. ${ }^{1,2}$ Because of their design, previous computer simulations did not allow for direct comparison of these quantities with experiment, and efficient technologies to exactly measure such defect densities in large-scale atomistic simulations did not even exist. Furthermore, previous MD computer studies that investigated the strengthening effect of nanotwins used almost exclusively columnar, quasi-twodimensional simulation geometries based on periodic boundary conditions. In addition to a very small number of grains, this implies a reduction in available slip systems as well as degrees of freedom for grain-boundary configurations. In fact, Frøseth et al. ${ }^{13}$ have demonstrated that a twodimensional simulation geometry can misleadingly promote mechanisms like deformation twinning in $\mathrm{Al}$, which are superseded by other mechanisms when using a more realistic, fully three-dimensional simulation setup. Particularly, in regard to dislocation nucleation, a fully three-dimensional mi- 


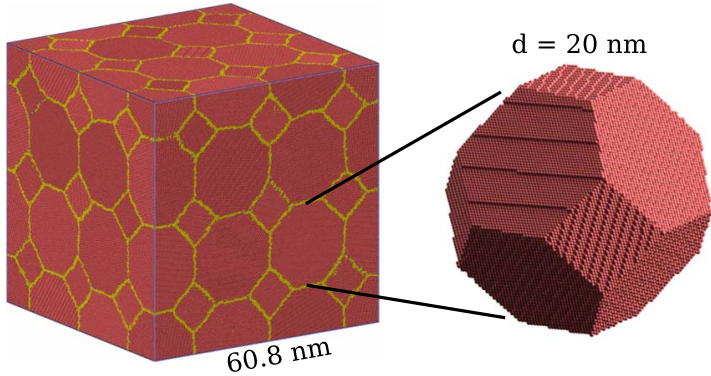

FIG. 1. (Color online) Nanocrystalline model structure used for MD simulations. The cubic simulation box contains 54 grains of the same size, having the same regular shape but different lattice orientations. The system contains approximately 15 million atoms and periodic boundary conditions were applied in all three spatial directions. Atoms in the grain boundaries have been marked with a yellow color.

crostructure is crucial for accurately describing inhomogeneous stress concentrations at nucleation sites, enabling the formation of curved dislocation segments, and to not preclude a multitude of possible reactions observed for dislocations on multiple slip systems.

In view of these results, the aim of this paper is to accurately compare the behavior of both nanotwinned $\mathrm{Cu}$ and $\mathrm{Pd}$ under realistic conditions and to assess whether the strengthening effect of twins observed in $\mathrm{Cu}$ is a general phenomenon present in all fcc metals. We use large-scale molecular dynamics simulations of nanotwinned and twin-free, $\mathrm{Cu}$ and $\mathrm{Pd}$ polycrystals to address this issue. The fully threedimensional model structures used in the present study consist of a statistically significant number of grains and grain boundaries. This allows for the formation of nonparallel dislocations lines and the inclusion of grain boundaries with both tilt and twist components in the microstructure without constraining the nucleation of dislocations. ${ }^{13}$ Moreover, we employ several newly developed analysis techniques ${ }^{26}$ for large-scale atomistic computer simulations of crystal plasticity. This allows us to extract the true dislocation, stacking fault, and TB densities as functions of strain from MD simulations and to directly compare them with experimental measurements.

\section{METHODS}

\section{A. Simulation procedure}

The Voronoi tessellation method was used to prepare three-dimensional, nanocrystalline model structures containing 54 grains with a size of $20.0 \mathrm{~nm}$. This relatively large grain size reduces the contribution of GB mechanisms, such as grain rotation and grain sliding to plasticity, which begin to dominate over dislocation glide at smaller grain sizes. ${ }^{27,28}$ The Voronoi points were positioned on a body-centered cubic superlattice, resulting in grains that have the shape of a truncated octahedron as shown in Fig. 1. Experimental structures often exhibit a log-normal grain-size distribution. Here, we used a single grain size and shape to enable a better comparison between different grain and twin orientations and different fcc materials, leaving all other preparation parameters the same. Periodic boundary conditions were applied in all three spatial directions to model a bulklike structure without free surfaces.

When defining the grain lattice orientations, we explicitly excluded low-angle grain boundaries and boundaries close to the $\Sigma 3$ twin configuration. In fact, the lattice orientation of each grain was repeatedly drawn from an isotropic random distribution until all grain boundaries in the structure satisfied two requirements. All grain-boundary misorientation angles were larger than $12^{\circ}$ and all as-prepared grain boundaries deviated from the twin configuration by more than $12^{\circ}$. Both constraints are necessary to avoid the coalescence of neighboring grains and the formation of coherent TBs due to grain rotation during the annealing phase described below.

In addition to the conventional nanocrystalline sample, which serves as a reference, the nanotwinned structure was prepared by introducing arrays of parallel twin planes with 4 nm spacing into each grain [see Fig. 3(a)]. This twin spacing was found by Li et al. ${ }^{15}$ to be the twin lamella thickness of maximum strength. Finally, we derived $\mathrm{Cu}$ structures from the Pd samples by rescaling the atomic coordinates by the ratio of the lattice constants, $a_{\mathrm{Cu}} / a_{\mathrm{Pd}}$, thereby preserving grain-boundary misorientations and other topological features. This procedure results in a slightly reduced grain size $(18.6 \mathrm{~nm})$ in the $\mathrm{Cu}$ samples, but at the same time, it ensures an equal number of atoms (and number of dislocation nucleation sites) in both structures. This enables a direct comparison of the microscopic dislocation processes in both fcc materials. Note that the crystallite size in all samples is too small to support conventional dislocation multiplication by Frank-Read mechanisms.

As shown by Vo et al., ${ }^{29}$ annealing of the samples at elevated temperatures is crucial for equilibrating grain boundaries in computer-generated polycrystals. Therefore all structures were annealed at $600 \mathrm{~K}$ for 400 ps before being quenched to $300 \mathrm{~K}$, at which all deformation experiments were performed. All structures prepared in this way contained only general, high-angle grain boundaries in addition to the growth twin arrays and were free of lattice dislocations.

MD simulations were performed with the molecular dynamics code LAMMPS. ${ }^{30}$ The atomic interactions were modeled with embedded-atom method potentials for palladium ${ }^{31}$ and copper, ${ }^{32}$ which correctly predict the experimental stacking-fault energies of the materials (see Fig. 8). We used Berendsen's thermostat and barostat ${ }^{33}$ to control temperature and relax the simulation cell size.

A strain-controlled, uniaxial tensile test at a constant engineering strain rate of $10^{8} \mathrm{~s}^{-1}$ up to $10 \%$ strain was conducted with each of the four samples. This corresponds to a simulation time of $1 \mathrm{~ns}$. During such a straining simulation, the box length is constantly increased in the tensile direction while the box size is allowed to relax in the two other directions. One has to keep in mind that the simulated strain rate is several orders of magnitude higher than typical experimental strain rates. This is an inherent limitation of every MD simulation but is necessary to obtain significant amount of deformation within a reasonable computation time. 


\section{B. Analysis techniques}

To visualize crystal defects (dislocations, stacking faults, grain boundaries, etc.), we employ the common neighbor analysis (CNA) method. ${ }^{34}$ The CNA is an established computational analysis method that characterizes the local crystalline order of each atom and thereby identifies defect atoms with a disordered arrangement of neighbors. The CNA alone, however, cannot distinguish between different types of crystal defects, nor can it determine any of their properties (like the Burgers vector of a dislocation). That is, it can only serve for visualization purposes. To overcome these limitations, we employ the on-the-fly dislocation detection algorithm (ODDA) (Ref. 26) to identify and characterize dislocation lines in the MD simulations. This algorithm transforms the atomistic dislocation cores into a line representation and determines their Burgers vectors. The resulting continuous dislocation lines enable a quantitative analysis of the geometric dislocation structure and dislocation reactions. The dislocation analysis is performed with a very fine-time resolution at intervals of 200 fs to yield the instantaneous line shape and Burgers vector of each dislocation segment in the sample during deformation. It also provides information on the glide system of dislocations and the junctions they form.

In addition, we have implemented a second, analysis method to distinguish intrinsic stacking faults (ISFs) from coherent TBs. In previous atomistic simulations, such planar defects were usually detected by identifying atoms having an hcp-like coordination, which is characteristic for both ISFs and TBs in fcc metals. Such hcp atoms can easily be extracted with the common neighbor analysis or similar methods alone. However, to distinguish between stacking faults and twin boundaries, an additional topological analysis of the surrounding atoms and crystal orientations is required. We have implemented such an improved analysis algorithm into the MD simulation code to count the respective numbers of atoms forming either ISFs or TBs. This, in turn, allows us to quantify the exact densities of stacking faults and twin boundaries in the material as a function of strain and to make ISFs and TBs easily distinguishable in the visualization pictures. In the following figures, ISFs are marked red and TBs blue, respectively.

Postprocessing and visualization of simulation snapshots were performed with the scientific software package Open Visualization Tool (OVITO), ${ }^{35}$ which is freely available.

\section{RESULTS}

The calculated stress-strain curve (Fig. 2) clearly shows that nanotwins strengthen the material in the case of $\mathrm{Cu}$. For $\mathrm{Pd}$, however, there is a clear softening effect due to the presence of twins. This difference is investigated in the following.

Figure 3 shows cross sections of the nanotwinned samples, revealing crystal defects formed inside the grains. Here, atoms belonging to coherent twin boundaries have been colored blue, intrinsic stacking faults have been marked red.

Nanotwinned $\mathrm{Cu}$ exhibits a very heterogeneous deformation behavior. Depending on the local grain and twin-

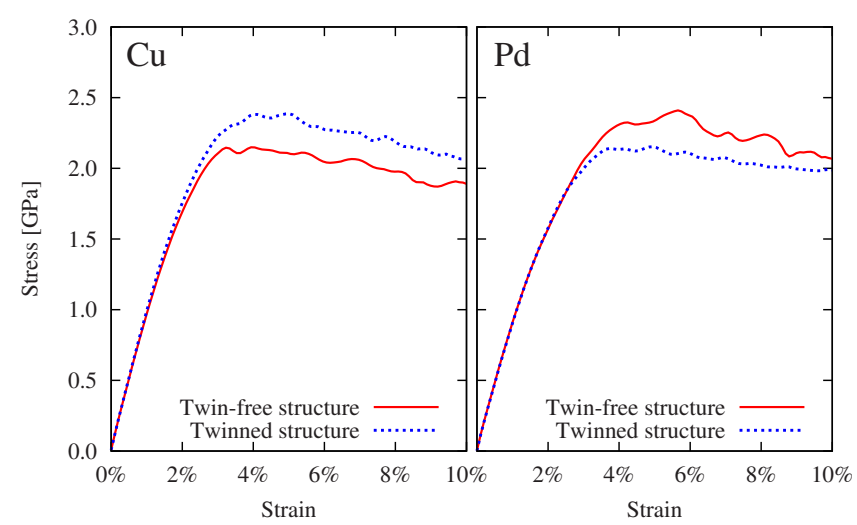

FIG. 2. (Color online) Stress-strain curves obtained for uniaxial tensile deformation of nanocrystalline $\mathrm{Cu}$ and $\mathrm{Pd}$. For each element, two different structures were tested: a defect-free microstructure with $20 \mathrm{~nm}$ grain size and an identical structure containing growth twins (4 nm spacing). The simulated strain rate was $10^{8} \mathrm{~s}^{-1}$.

boundary orientation, we observe a combination of one or more of the following dislocation mechanisms: twinboundary migration, emission of partial or perfect lattice dislocations from the grain boundaries, formation of extrinsic stacking faults, and interaction of partial dislocations with twin boundaries. These processes have been described before for MD simulations of nanotwinned copper. ${ }^{14,18}$ In several grains, we observe dislocation transmission through twin boundaries. However, the contribution of these transmitted dislocations to the overall plasticity is not significant. The formation of complex, three-dimensional dislocation entanglements can be observed. Partial dislocation segments are strongly curved, and at later stages of the deformation all sets of glide planes become populated in some of the nanotwinned grains.

A few of the randomly oriented grains in the simulated structure contain TBs with a high Schmid factor orientation. Dislocations in these grains are nucleated on slip systems parallel to the twins and no intersection of dislocations with the TB occurs. In contrast to these grains, grains with TBs on low Schmid factor planes show frequent blocking of transverse dislocations at the TBs. Partial dislocation loops, being nucleated from a grain boundary, are usually confined in between a single twin lamella.

In contrast, nanotwinned Pd deforms almost exclusively via twin-boundary migration. Irrespective of the local grain and $\mathrm{TB}$ orientation, primarily twinning dislocations are nucleated on glide planes parallel and adjacent to TBs. This restriction of dislocations to a single set of glide planes per grain, which mostly have low Schmid factors, demands an increased dislocation activity to reach a certain level of tensile strain. As can be seen in Fig. 3(c), the twinning partials form a two-dimensional network on the twin planes. In several cases, we observe pile-ups of consecutive twinning partials. Even though dislocation slip is restricted to a single set of planes per grain, the polycrystalline microstructure does not fail. Instead, incompatible deformations of the grains are accommodated by GB motion and atomic shuffling in the GBs. In Fig. 3(c), the displaced GB structure is marked with a yellow color. 

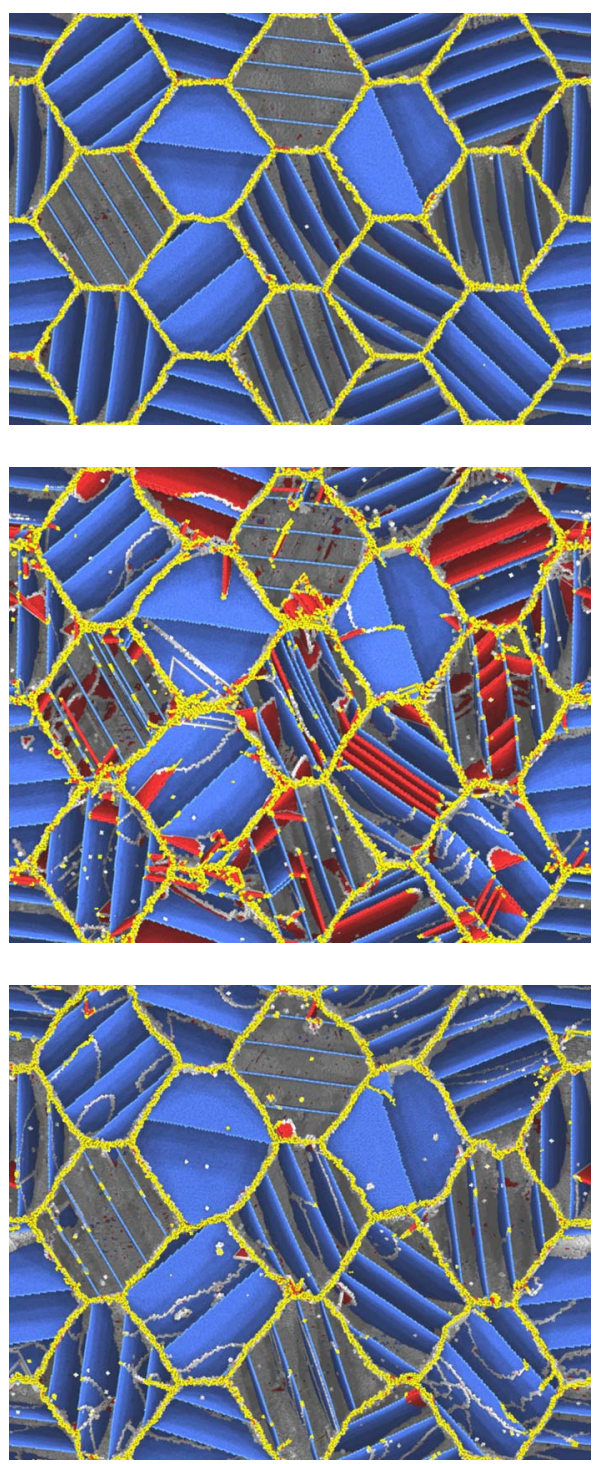

FIG. 3. (Color online) Slices through the [110] plane of the simulation box showing the interior of the nanotwinned grains. Crystal atoms with a perfect fcc environment have been removed in these pictures. (a) The as-prepared structure with regular-spaced twin boundaries in randomly oriented $20 \mathrm{~nm}$ grains. (b) Twinned nc-Cu after $10 \%$ tensile strain (along vertical axis). (c) Twinned nc-Pd after $10 \%$ tensile strain. Stacking faults have been marked red and coherent twin boundaries blue.

\section{A. Planar fault densities}

We have measured the densities of intrinsic stacking faults and coherent twin boundaries that develop during deformation (third row of Fig. 4). The conventional nanocrystalline $\mathrm{Cu}$ and $\mathrm{Pd}$ samples are free of any planar faults in the beginning. The first plot in row three of Fig. 4 shows, however, that deformation twinning is an active mechanism in nanocrystalline $\mathrm{Cu}$, which sets in at $3.4 \%$ strain. A considerable number of TBs (blue color) is generated during deformation (reaching a density of $0.9 \times 10^{8} \mathrm{~m}^{-1}$ after $10 \%$ strain). Deformation twinning is preceded by stacking fault formation starting at approximately $2 \%$ strain. After $10 \%$ strain, an ISF density of $0.9 \times 10^{8} \mathrm{~m}^{-1}$ is reached. In nc-Pd, the generated number of planar faults is much smaller: Only about $0.06 \times 10^{8} \mathrm{~m}^{-1}$ ISF and TB content is measured after $10 \%$ strain. These planar defects are found in slightly dissociated perfect dislocations and debris structures as shown in Fig. 7(b).

The structures with as-prepared growth twins initially contain a TB density of $2.3 \times 10^{8} \mathrm{~m}^{-1}$, which roughly corresponds to the $4 \mathrm{~nm}$ twin lamella spacing. In nanotwinned $\mathrm{Cu}$, this density stays almost constant during deformation, while intrinsic stacking faults are generated by leading partials reaching a density of $0.6 \times 10^{8} \mathrm{~m}^{-1}$. In nanotwinned $\mathrm{Pd}$, the TB density decreases slightly during deformation as TBs loose coherency when more and more twinning partials are accumulated.

\section{B. Dislocation densities}

We employed the dislocation detection algorithm ODDA (Ref. 26) to extract all lattice and twin-boundary dislocation segments in the MD simulations and to measure their Burgers vector and length. The overall dislocation density can be directly derived from this data. One of the peculiarities of the ODDA is that it detects dislocations forming small-angle GBs and vicinal twin boundaries when a GB dissociates into discrete dislocation cores. Even though we explicitly avoided these types of GBs in our as-prepared structures, structural and angular changes in the GB during the deformation are inevitable. Thus, to suppress the detection of immobile GB dislocations, we excluded a $0.7-\mathrm{nm}$-wide region on each side of the geometric grain-boundary planes from our analysis, thereby taking only lattice dislocations in the grain interiors into account.

In case of dissociated $(a / 2)\langle 110\rangle$ dislocations, the analysis algorithm yields in most cases the leading and trailing Shockley partials separately. A very small separation distance of one or two times the nearest-neighbor spacing is sufficient to resolve the cores of the two partials. ${ }^{26}$

Lattice Shockley partials and twinning partials in fcc crystals have the same $(a / 6)\langle 112\rangle$ type of Burgers vector, making them indistinguishable if only this vector is taken into account. However, they play different roles in plastic deformation. The former dislocation type carries conventional dislocation slip, whereas the latter type takes part in twinboundary migration. Thus, to distinguish these two types of partial dislocations, we count the number of crystallites through which a Burgers circuit passes when it is traced around the dislocation core: The core of a lattice Shockley partial is embedded in a single crystallite whereas a twinning partial has a Burgers vector parallel to the TB and is adjacent to two crystallites having the characteristic twin configuration.

Figure 4 (second row) shows the measured dislocation densities (averaged over all grains) as a function of tensile strain, $\epsilon$. The total dislocation density, $\rho$, is split up into contributions from lattice Shockley partials, $\rho_{\text {lat }}$, twinning partials, $\rho_{t w i n}$, and other dislocations $[\vec{b} \neq(a / 6)\langle 112\rangle], \rho_{\text {other }}$. Beginning at a strain level of $\epsilon=1.92 \%$, the dislocation density in initially twin-free $\mathrm{nc}-\mathrm{Cu}$ increases almost linearly to $\rho=2.8 \times 10^{16} \mathrm{~m}^{-2}$ after $10 \%$ strain. This includes a consid- 

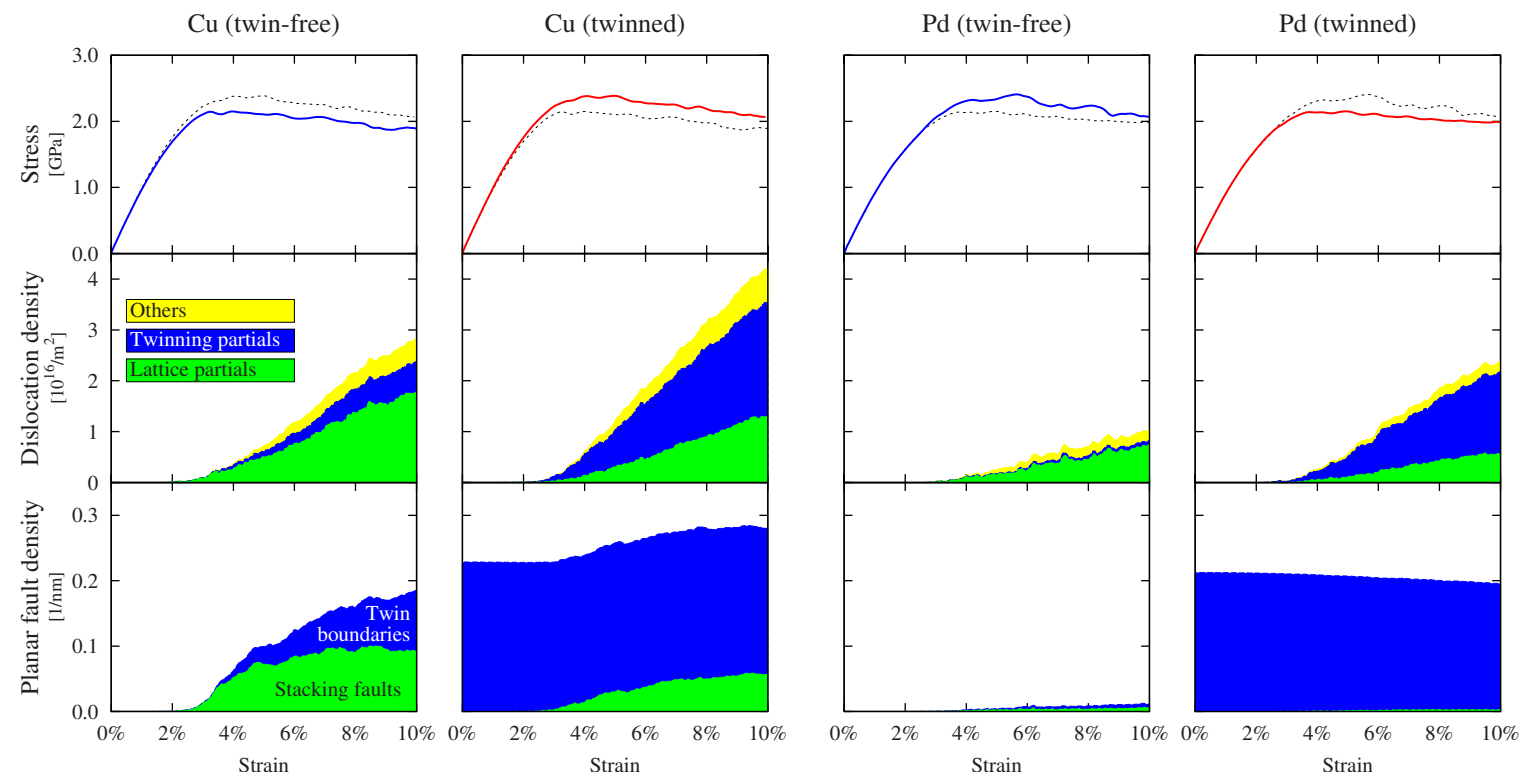

FIG. 4. (Color online) Measured tensile stress (first row), dislocation density (second row), and planar fault density (third row) in simulated tensile tests as functions of strain.

erable number of twinning partials, reflecting the generation of deformation twins in $\mathrm{nc}-\mathrm{Cu}$. In nc-Pd, the accumulation of dislocations is smaller. Only a density of $\rho=1.0 \times 10^{16} \mathrm{~m}^{-2}$ is reached in this material. Less than $8 \%$ of this value are twinning dislocations.

The introduction of growth twins promotes the accumulation of dislocations in both materials. Up to $\rho=4.2$ $\times 10^{16} \mathrm{~m}^{-2}$ of total dislocation density is reached in nanotwinned $\mathrm{Cu}$ after deformation. The density of twinning partial reaches a level of $\rho_{t w i n}=2.2 \times 10^{16} \mathrm{~m}^{-2}$. In nanotwinned Pd, the measured dislocation density is $\rho=2.4 \times 10^{16} \mathrm{~m}^{-2}$, with $\rho_{\text {twin }}=1.6 \times 10^{16} \mathrm{~m}^{-2}$ contributed by twinning dislocations.

\section{DISLOCATION PLASTICITY IN NANOCRYSTALLINE COPPER AND PALLADIUM}

The reference simulations of twin-free, conventional nanocrystalline $\mathrm{Cu}$ and $\mathrm{Pd}$ provide some interesting insights into the role of grain boundaries as dislocation sources. Figure 5 shows cross sections of the nanocrystalline samples after deformation.

To study the onset of dislocation plasticity in the nanocrystalline structures, we use the dislocation detection algorithm to monitor all grains in the structure for the nucleation of dislocations. In twin-free $\mathrm{Cu}$, the first leading partial dislocation is nucleated from a GB triple junction at $1.92 \%$ tensile strain. The Schmid factor for the corresponding $\{111\}\langle 112\rangle$ slip system is 0.496 , which is the fifth largest among all 648 slip systems in the polycrystalline model structure. The nucleation of this first partial is immediately followed by the depinning of an already existing partial in another grain at $2.02 \%$ tensile strain. Figure 6 shows this depinning process, with the partial being part of an array of dislocation embryos formed during GB relaxation at the beginning of the simulation. The Schmid factor of the corresponding slip system is only 0.434 (position 63 in the
Schmid factor list), exemplifying that the structure of the grain boundary is a very important factor that can greatly enhance dislocation nucleation.

As the deformation proceeds, other grain boundaries begin to emit dislocations as well. The low stacking-fault en-
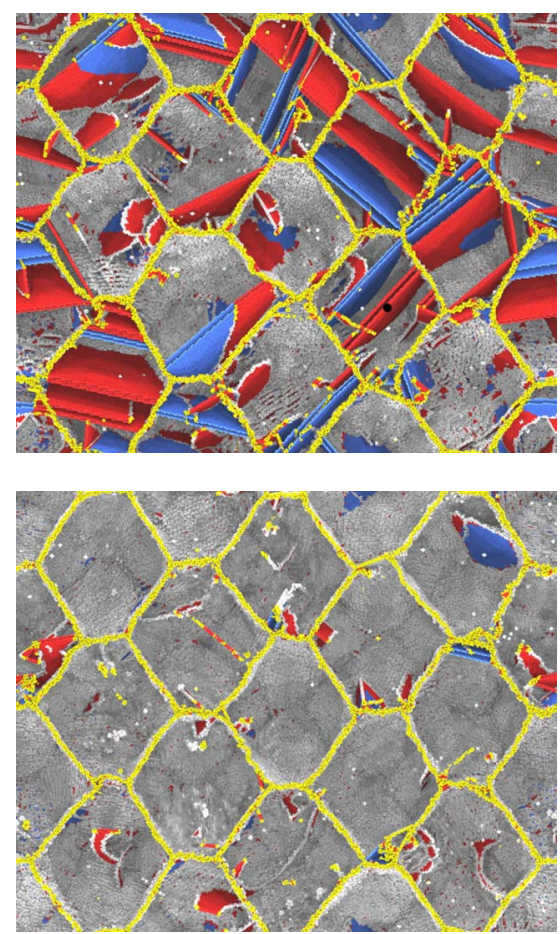

FIG. 5. (Color online) Slices through the [110] plane of the simulation box showing the interior of the grains. Crystal atoms with a perfect fcc environment have been removed to reveal grain boundaries, dislocations, and planar faults. (a) Nanocrystalline $\mathrm{Cu}$ (20 nm grain size) after $10 \%$ tensile strain (along vertical axis). (b) Nanocrystalline Pd after 10\% tensile strain. Stacking faults have been marked red and coherent twin boundaries blue. 

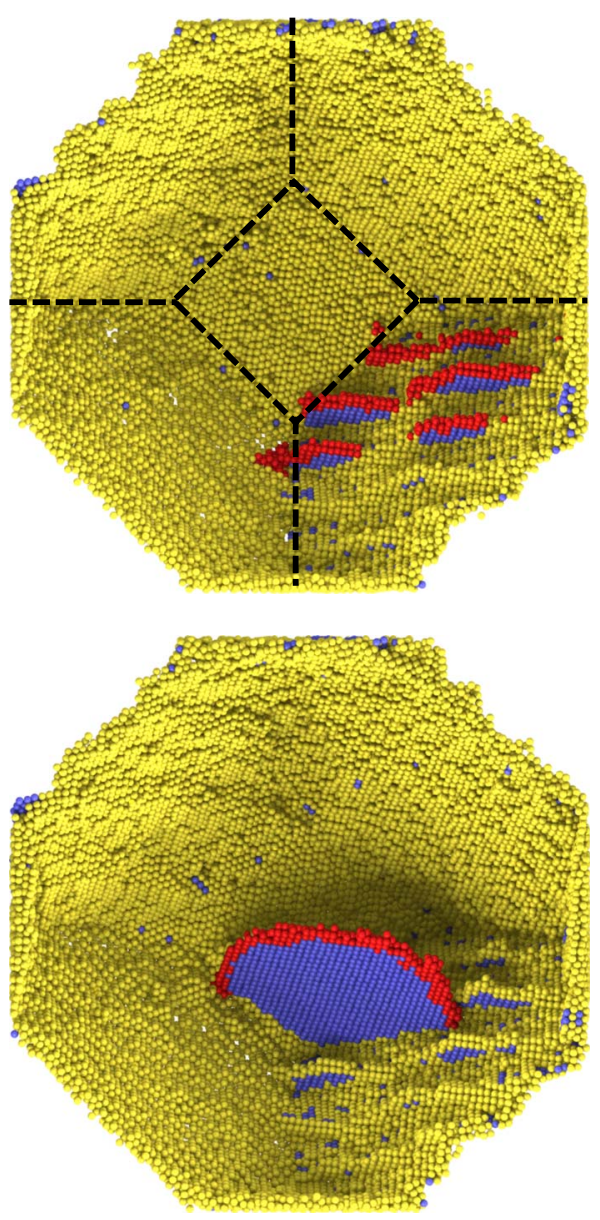

FIG. 6. (Color online) Two snapshots of a grain boundary as seen from inside the grain. (a) The lower right part of the GB has a particular misorientation angle that results in the formation of an array of dislocation embryos during relaxation of the GB (marked red). Dashed lines denote GB triple junctions. (b) After loading the sample one of these embryos is activated and evolves to a lattice dislocation loop. In both $\mathrm{Cu}$ and $\mathrm{Pd}$, this is one of the very first dislocation events, which occurs before other slip systems with higher Schmid factors are activated.

ergy of $\mathrm{Cu}$ results in a splitting distance larger than the grain size, i.e., stacking faults span whole grains as being evident in Fig. 5(a). We observe frequent generation of deformation twins. That is, intrinsic stacking faults left behind by leading partials are, in some cases, first transformed into extrinsic stacking faults, which then eventually widen to nanosized twin lamellae. This observation is confirmed by the increasing twin-boundary density that we measure in the nanocrystalline $\mathrm{Cu}$ structure (lower left plot in Fig. 4). Twin growth proceeds by the emission of twinning dislocations on a parallel crystal plane adjacent to the TB.

In nc-Pd, the first observed dislocation is again the very same pre-existing partial dislocation that was formed during the relaxation of a grain boundary (Fig. 6) and was present in the $\mathrm{Cu}$ sample as well. It unpins at $2.70 \%$ strain, andtypical for $\mathrm{Pd}$ - is immediately followed by a trailing partial. During incipient dislocation activity, most dislocations reach the opposite grain boundary unobstructedly. Simultaneously, however, Lomer dislocations are nucleated at several grain
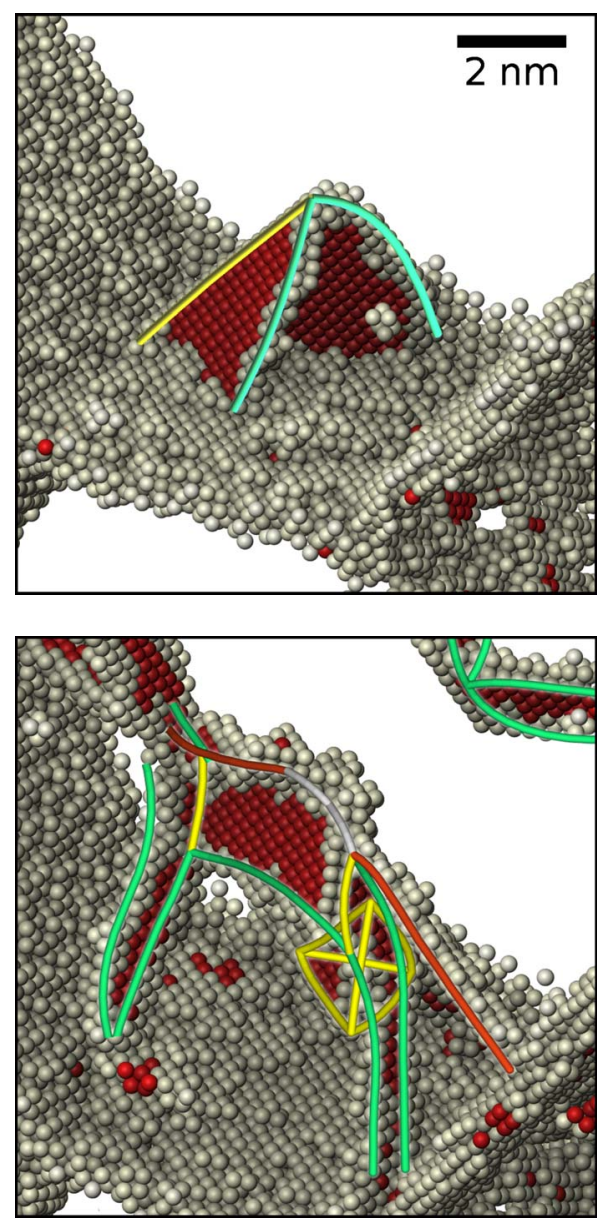

FIG. 7. (Color online) Example for a Lomer dislocation nucleated from a grain boundary in nanocrystalline Pd. The automatically extracted dislocation segments have been superimposed on the atomistic visualization. The left picture shows the stair-rod dislocation (yellow line) and the two stacking faults at an initial stage right after nucleation (at $4.3 \%$ strain). The right picture shows the same part of the crystal after $8.3 \%$ strain. After grown further, the Lomer dislocation has undergone several reactions with other dislocations and a complex defect structure has formed, including a stackingfault tetrahedron (made of four yellow lines). Shockley partials are represented by green lines and perfect dislocations by red lines.

boundaries and slowly grow into the interior of the grains. Figure 7(a) exemplarily shows such a dislocation. They act as obstacles to dislocations, and often, complex reactions with other dislocations can be observed. Such reactions leave immobile dislocation debris behind, which contribute to the measured dislocation density (Fig. 4) and lead to an accumulation of dislocations in the grain interior [Fig. 7(b)].

\section{DISCUSSION}

From the measured stress-strain curves (Fig. 2), we find that nanotwins strengthen the material in the case of $\mathrm{Cu}$, whereas they have a softening effect in $\mathrm{Pd}$. As stated in the introduction, twin boundaries can function in two ways: being barriers for dislocation propagation and acting as dislocation sources. The latter effect dominates in nanotwinned 


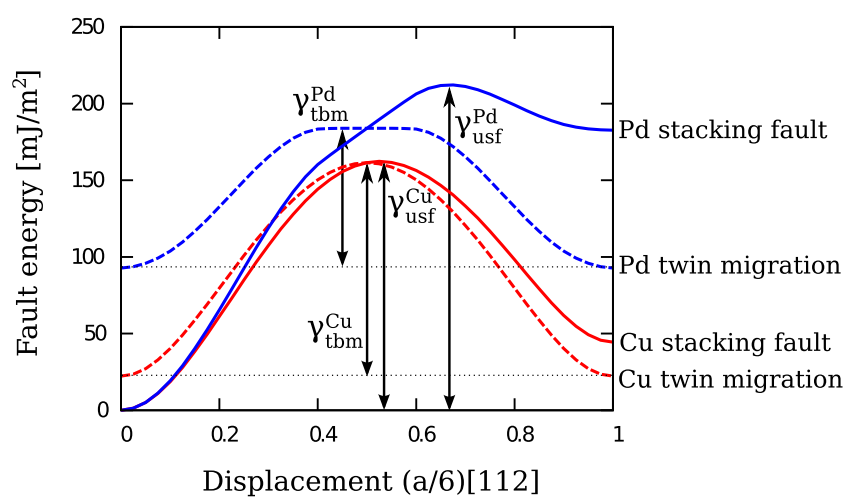

FIG. 8. (Color online) GPF energy curves for $\mathrm{Cu}$ and $\mathrm{Pd}$. The ratio of unstable stacking-fault energy, $\gamma_{\text {usf }}$, to twin-boundary migration energy, $\gamma_{\mathrm{tbm}}$, is close to unity for $\mathrm{Cu}$. In $\mathrm{Pd}$, twin-boundary migration is energetically favored over stacking-fault formation.

$\mathrm{Pd}$, where we observe a 21-fold increase in twinning partials and a 2.6-fold total increase in dislocations caused by the growth twins. In contrast, the total density of both lattice and twinning partial dislocations in $\mathrm{Cu}$ is increased by only $50 \%$ due to the twins. The density of lattice partials slightly decreases in both metals when growth twins are present, because loop expansion is blocked by twin boundaries (found for $\mathrm{Cu}$ ), or because nucleation of twinning partials is more favorable (found for $\mathrm{Pd}$ ).

To better understand the differences between nanotwinned $\mathrm{Cu}$ and $\mathrm{Pd}$, we have calculated the generalized planar fault (GPF) energy curves for stacking fault formation and twinboundary migration in both metals (Fig. 8). The relative heights of the energy barriers associated with the nucleation of a lattice partial (being the generator of stacking faults) and a twinning partial (carrying twin-boundary migration) can be estimated from these curves. For $\mathrm{Cu}$, both nucleation barriers have a similar height, whereas Pd shows a twin migration energy barrier that is much smaller than the stacking-fault nucleation barrier. That is, the nucleation of twinning partials gliding parallel to the twin planes is energetically favored in $\mathrm{Pd}$, and growth twins can no longer act as obstacles to dislocation motion in this case. Frøseth et al. have found a similar behavior for nanotwinned $\mathrm{Al}$ and give an in-depth discussion of this effect. ${ }^{13}$

Our dislocation analysis algorithm allows us to determine the glide system of each partial dislocation nucleated during deformation. From this data, highly populated glide systems can be identified. Figure 9 displays the density of partial dislocations as a function of Schmid factor in the polycrystal. As expected, in twin-free $\mathrm{Cu}$ and $\mathrm{Pd}$, the majority of dislocations occur on slip systems having high Schmid factors. Here, one must pay attention to the fact that the statistical number of available slip systems with a high Schmid factor is much smaller than the number of systems with a low Schmid factor, given a polycrystal with random grain orientations (dashed curve in Fig. 9). In the presence of growth twins, the difference between $\mathrm{Cu}$ and $\mathrm{Pd}$ becomes evident: In nanotwinned $\mathrm{Cu}$, most dislocations accumulate on glide systems with a high Schmid factor, irrespective of the orientation of the twins. In nanotwinned $\mathrm{Pd}$, the glide system population is shifted to lower Schmid factors and resembles the
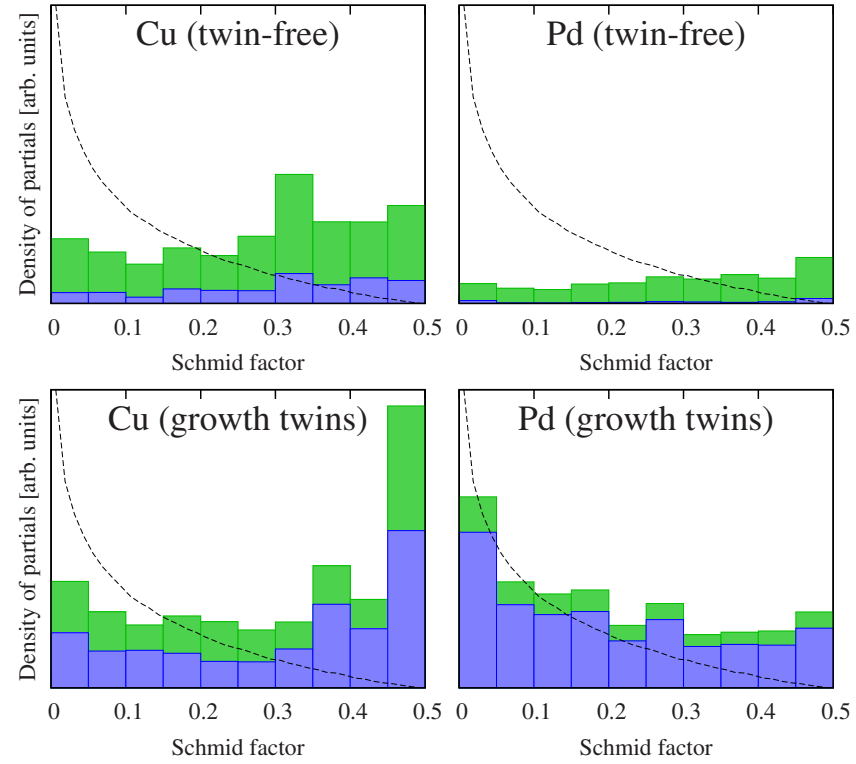

FIG. 9. (Color online) Average content of partial dislocations broken down into Schmid factor levels. These histograms have been generated by determining the Schmid factor of the slip system of each partial dislocation segment in the polycrystal. Blue bars denote contributions of twinning partials. For reference, the dashed curve indicates the number of available slip systems in an fcc polycrystal with random grain orientations.

statistical orientation distribution of the twin planes.

This evidences that in nanotwinned Pd, the low twin migration barrier dictates the glide planes on which the majority of dislocations is nucleated. That is, the Schmid factor no longer influences the activation of slip systems. In nanotwinned $\mathrm{Cu}$, which exhibits a TB migration energy comparable to the stacking-fault nucleation barrier, the deformation mode of a grain primarily depends on its relative orientation to the tensile axis. If TBs are parallel to planes with a high Schmid factor, dislocation glide occurs along the TBs and dislocations are not blocked. If, however, the grain orientation promotes dislocation nucleation on transverse glide planes, then TBs act as obstacles and have a strengthening effect (Fig. 10). Shabib and Miller ${ }^{19}$ proposed a composite materials model with grains of the "ductile" phase and of the "strengthening" phase to characterize such an inhomogeneous behavior.

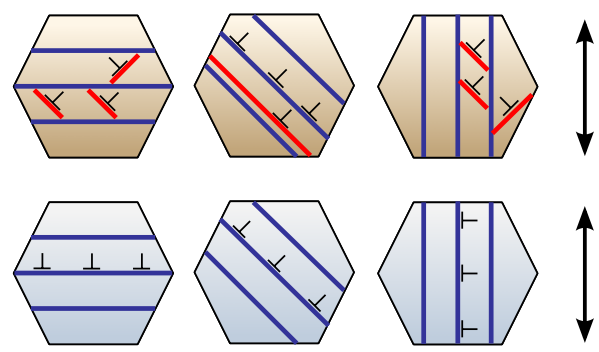

FIG. 10. (Color online) Schematic illustration of the preferred glide systems in grains with varying orientation in nanotwinned $\mathrm{Cu}$ and Pd. Blue lines are pre-existing growth twins, red lines represent intrinsic stacking faults generated by Shockley partials. 
For deformed nanotwinned copper, experimental results indicate high densities of stacking faults and Shockley partials associated with TBs. ${ }^{1-3}$ Our simulations confirm these qualitative observations and provide dislocation densities as a function of strain. The density of twinning partials reaches $2.2 \times 10^{16} \mathrm{~m}^{-2}$ in nanotwinned $\mathrm{Cu}$ after deformation. This is in agreement with an estimation made by Lu et al. ${ }^{3}$ who report a twinning partial density of $5 \times 10^{16} \mathrm{~m}^{-2}$ determined from TEM images of nanotwinned $\mathrm{Cu}$ with $4 \mathrm{~nm}$ twinboundary spacing.

In initially twin-free $\mathrm{Cu}$, we observe deformation twinning (accompanied by an increasing twin-boundary density, see first plot in third row of Fig. 4), which is suppressed in pretwinned $\mathrm{Cu}$. Our simulations predict, however, a considerable generation of stacking faults in nanotwinned $\mathrm{Cu}$. We measure a final ISF density of $0.6 \times 10^{8} \mathrm{~m}^{-1}$ after deformation. This is in qualitative agreement with experimental observations, ${ }^{1,3}$ though, reliable experimental numbers have not been published.

In all four simulations, we measure a decreasing flow stress with increasing tensile strain (Fig. 2). This observation is in line with other computational studies. Brandl et al. ${ }^{36}$ rationalized this by the occurrence of cross slip, which allows dislocations to avoid regions of back stresses in the grain boundaries caused by preceding dislocations not being fully absorbed in the boundaries. We, however, do not observe any significant occurrence of cross slip in our simulations of nanocrystalline $\mathrm{Cu}$ and $\mathrm{Pd}$. Instead, we can report a constantly increasing density of dislocations in the material (second row of Fig. 4), which certainly promotes plastic slip at higher strains. Our dislocation analysis gives no information on the mobility of dislocations, i.e., the exact fraction of pinned dislocations remains unknown. More advanced analysis methods need to be developed first to determine the contribution of individual dislocation lines to the total plastic slip.

The role of certain grain boundaries as effective dislocation sources is another important aspect that was revealed by our simulations. When preparing our virtual test samples, we explicitly excluded vicinal $\Sigma 3 \mathrm{TBs}$ as well as low-angle GBs. Both types of grain boundaries are already known to be effective sources for lattice dislocations. ${ }^{37,38}$ Our simulations of nanocrystalline $\mathrm{Cu}$ and $\mathrm{Pd}$ show, however, that other highangle grain boundaries can also exhibit a partially ordered structure. During relaxation of such as-prepared grain boundaries, an array of dislocation embryos evolves (see Fig. 6). These stacking faults can reach several angstroms into the grains. During deformation, they act as dislocation sources, which can easily be activated because no nucleation barrier has to be overcome. That is, our results show that some general high-angle grain boundaries can serve as efficient dislocation sources, similar to vicinal twin boundaries, and thereby promote the activation of nonoptimal slip systems at low stresses.

Li et al. ${ }^{24}$ have performed simulations of nanotwinned $\mathrm{Cu}$ with varying twin-boundary spacings ranging from $\lambda=0.63$ to $6.25 \mathrm{~nm}$. Their simulations show that there exists an optimal twin spacing with a highest strength that is grain size dependent. For a grain size of $d=20 \mathrm{~nm}$, similar to the grain size used in our simulations, they find a decreasing flow stress in nanotwinned $\mathrm{Cu}$ in the twin spacing range below $\lambda=2 \mathrm{~nm}$. Note, however, that even at the highest twin density, the observed strength never falls below the strength of twin-free, nanocrystalline $\mathrm{Cu}$ corresponding to an infinite twin spacing, that is, pre-existing twin boundaries always provide at least some strengthening effect in $\mathrm{Cu}$. In $\mathrm{Pd}$, in contrast, we find a real softening effect of twin boundaries at an intermediate twin spacing of $\lambda=4 \mathrm{~nm}$. Taking into account the mechanism that is responsible for this softening, one can conclude that a strengthening effect of twin boundaries in Pd may only exist at much larger grain sizes, which allow for a larger twin spacing.

\section{SUMMARY}

We have performed large-scale molecular-dynamics simulations of nanotwinned fcc materials to study the effect of coherent twin boundaries on mechanical strength under uniaxial tension. All test samples consisted of a statistically significant number of grains and were prepared in exactly the same way to ensure comparability between materials as well as between twinned and conventional microstructures. A fully three-dimensional simulation setup was used to avoid intrinsic limitations of quasi-two-dimensional simulation cells. Our simulations clearly show that twin boundaries lower the yield strength of $\mathrm{Pd}$, in contrast to nanotwinned $\mathrm{Cu}$, were a strengthening effect is observed.

Based on a dislocation analysis method that identifies dislocation lines in atomistic simulations, we report exact dislocation densities in nanotwinned $\mathrm{Cu}$ and $\mathrm{Pd}$ as well as in their conventional nanocrystalline counterparts as a function of strain. Our measurements agree well with experimental data given for nanotwinned $\mathrm{Cu}$. To explain the softening effect of twins in $\mathrm{Pd}$, we determined the dominating type of dislocations in the material by partitioning the total dislocation density into contributions from lattice dislocations and twinning partials. We find that the low twin migration energy barrier of Pd promotes the generation of twinning partials at twin boundary-grain-boundary junctions.

In nanotwinned $\mathrm{Cu}$, dislocations are preferentially nucleated on slip systems with high Schmid factors. This leads to a blocking of dislocations in grains with twin boundaries on transversal crystal planes and the observed strengthening effect. In nanotwinned $\mathrm{Pd}$, dislocations are restricted to twinboundary planes, irrespective of their Schmid factor, and plastic deformation proceeds by unhindered twin-boundary migration.

\section{ACKNOWLEDGMENTS}

The authors acknowledge the financial support of the Deutsche Forschungsgemeinschaft (FOR714) and the grants for computing time by the Forschungszentrum Jülich and bwGRiD, member of the German D-Grid initiative. D.F. acknowledges support from DOE, Basic Energy Sciences. K.A. is grateful for the hospitality experienced at Virginia Tech during his sabbatical leave. 
*stukowski@mm.tu-darmstadt.de

${ }^{1}$ L. Lu, R. Schwaiger, Z. Shan, M. Dao, K. Lu, and S. Suresh, Acta Mater. 53, 2169 (2005).

${ }^{2}$ M. Dao, L. Lu, Y. F. Shen, and S. Suresh, Acta Mater. 54, 5421 (2006).

${ }^{3}$ L. Lu, X. Chen, X. Huang, and K. Lu, Science 323, 607 (2009).

${ }^{4}$ K. Lu, L. Lu, and S. Suresh, Science 324, 349 (2009).

${ }^{5}$ L. Lu, Y. Shen, X. Chen, L. Qian, and K. Lu, Science 304, 422 (2004).

${ }^{6}$ Z. S. You, L. Lu, and K. Lu, Scr. Mater. 62, 415 (2010).

${ }^{7}$ E. Qin, L. Lu, N. Tao, J. Tan, and K. Lu, Acta Mater. 57, 6215 (2009).

${ }^{8}$ L. Lu, T. Zhu, Y. Shen, M. Dao, K. Lu, and S. Suresh, Acta Mater. 57, 5165 (2009).

${ }^{9}$ C. J. Shute, B. D. Myers, S. Xie, T. W. Barbee, Jr., A. M. Hodge, and J. R. Weertman, Scr. Mater. 60, 1073 (2009).

${ }^{10}$ A. M. Hodge, Y. M. Wang, and T. W. Barbee, Jr., Mater. Sci. Eng., A 429, 272 (2006).

${ }^{11}$ A. Hodge, Y. Wang, and T. Barbee, Jr., Scr. Mater. 59, 163 (2008).

${ }^{12}$ Y. B. Wang, B. Wu, and M. L. Sui, Appl. Phys. Lett. 93, 041906 (2008).

${ }^{13}$ A. G. Frøseth, H. Van Swygenhoven, and P. M. Derlet, Acta Mater. 52, 2259 (2004).

${ }^{14}$ A. G. Frøseth, P. M. Derlet, and H. V. Swygenhoven, Appl. Phys. Lett. 85, 5863 (2004).

${ }^{15}$ L. Li and N. M. Ghoniem, Phys. Rev. B 79, 075444 (2009).

${ }^{16}$ Z. X. Wu, Y. W. Zhang, and D. J. Srolovitz, Acta Mater. 57, 4508 (2009).

${ }^{17}$ Y. Kulkarni and R. J. Asaro, Acta Mater. 57, 4835 (2009).

${ }^{18}$ I. Shabib and R. E. Miller, Acta Mater. 57, 4364 (2009).

${ }^{19}$ I. Shabib and R. E. Miller, Modell. Simul. Mater. Sci. Eng. 17, 055009 (2009).

${ }^{20}$ A. J. Cao and Y. G. Wei, J. Appl. Phys. 102, 083511 (2007).

${ }^{21}$ Z.-H. Jin, P. Gumbsch, E. Ma, K. Albe, K. Lu, H. Hahn, and H. Gleiter, Scr. Mater. 54, 1163 (2006).

${ }^{22}$ Z.-H. Jin, P. Gumbsch, K. Albe, E. Ma, K. Lu, H. Gleiter, and H. Hahn, Acta Mater. 56, 1126 (2008).

${ }^{23}$ H. Zhou and S. Qu, Nanotechnology 21, 035706 (2010).

${ }^{24}$ X. Li, Y. Wei, L. Lu, K. Lu, and H. Gao, Nature (London) 464, 877 (2010).

${ }^{25}$ A. P. Sutton and R. W. Baluffi, Interfaces in Crystalline Materials (Clarendon, Oxford, 1995).

${ }^{26}$ A. Stukowski and K. Albe, Modell. Simul. Mater. Sci. Eng. 18, 025016 (2010).

${ }^{27}$ N. Q. Vo, R. S. Averback, P. Bellon, S. Odunuga, and A. Caro, Phys. Rev. B 77, 134108 (2008).

${ }^{28}$ J. Schiotz and K. W. Jacobsen, Science 301, 1357 (2003).

${ }^{29}$ N. Q. Vo, R. S. Averback, P. Bellon, and A. Caro, Phys. Rev. B 78, 241402(R) (2008).

${ }^{30}$ S. Plimpton, J. Comput. Phys. 117, 1 (1995).

${ }^{31}$ S. M. Foiles and J. J. Hoyt, Sandia National Laboratories Technical Report No. SAND2001-0661, 2001 (unpublished).

${ }^{32}$ Y. Mishin, M. J. Mehl, D. A. Papaconstantopoulos, A. F. Voter, and J. D. Kress, Phys. Rev. B 63, 224106 (2001).

${ }^{33}$ H. J. C. Berendsen, J. P. M. Postma, W. F. van Gunsteren, A. DiNola, and J. R. Haak, J. Chem. Phys. 81, 3684 (1984).

${ }^{34}$ J. D. Honeycutt and H. C. Andersen, J. Phys. Chem. 91, 4950 (1987).

${ }^{35}$ A. Stukowski, Modell. Simul. Mater. Sci. Eng. 18, 015012 (2010).

${ }^{36}$ C. Brandl, P. M. Derlet, and H. Van Swygenhoven, Philos. Mag. 89, 3465 (2009).

${ }^{37}$ A. G. Froseth, P. M. Derlet, and H. Van Swygenhoven, Scr. Mater. 54, 477 (2006).

${ }^{38}$ D. Farkas and L. Patrick, Philos. Mag. 89, 3435 (2009). 